\title{
GESTÃO ESTRATÉGICA DO CONHECIMENTO: UMA PROPOSTA DE FORMAÇÃO PROFISSIONAL
}

\author{
Ana Paula Lisboa Sohn, ${ }^{1}$ Filipa Dionísio Vieira, ${ }^{2}$ Nelson Casarotto Filho, ${ }^{3}$ \\ João Artur de Souza ${ }^{4}$ \\ RESUMO
}

O presente artigo tem o objetivo de desenvolver uma proposta de formação profissional, no nível de Especialização, voltada para atender às demandas dos profissionais do conhecimento. Para o alcance do objetivo proposto, faz-se uma análise sobre a demanda pelas organizações de profissionais que desenvolvam processos relacionados à gestão do conhecimento. Apresenta-se uma discussão sobre habilidades necessárias ao profissional envolvido com a gestão estratégica do conhecimento. Traça-se, ainda, um panorama dos cursos de formação para os profissionais do conhecimento. E, a partir de uma análise das informações levantadas, é desenvolvida a matriz curricular do curso proposto. Recomenda-se que a matriz curricular envolva questões relacionadas a habilidades conceituais, interpessoais e técnicas. Destaca-se, por fim, a importância de um diálogo frequente entre as organizações e a academia, para que os cursos ofertados venham ao encontro das necessidades demandadas pelo mercado.

Palavras-chave: profissional do conhecimento; gestão estratégica do conhecimento; formação profissional.

\section{STRATEGIC KNOWLEDGE MANAGEMENT: A PROPOSAL FOR TRAINING}

\begin{abstract}
This article aims to develop a proposal for professional training as a post-degree course geared to meet the demands of knowledge workers. To achieve the proposed objective an analysis on the demand for professionals who develop processes related to knowledge management was made. It presents a discussion on the necessary skills by the professional involved with the strategic knowledge management. An overview of training courses aimed at knowledge worker is drawn and, from an analysis of the collected information, the curriculum of the proposed course is developed. It is recommended that the curriculum involves issues related to conceptual, interpersonal and technical skills. Finally, it is emphasized the importance of a regular dialogue between organizations and academia to ensure that the courses offered will meet the needs of demands by organizations.
\end{abstract}

Keywords. knowledge worker; knowledge management; professional training.

\footnotetext{
Universidade Federal de Santa Catarina, Programa de Pós Graduação em Engenharia de Produção. Campus Universitário, Florianópolis - SC; anasohn@hotmail.com

Universidade do Minho, Departamento de Produção e Sistemas. Campus de Azurém, Guimarães, Portugal; filipadv@dps.uminho.pt

Universidade Federal de Santa Catarina, Programa de Pós Graduação em Engenharia de Produção. Campus Universitário, Florianópolis - SC, Brasil; nelson.casarotto@ufsc.br

4 Universidade Federal de Santa Catarina, Programa de Pós Graduação em Engenharia do Conhecimento. Campus Universitário, Florianópolis SC; jartur@gmail.com
} 


\section{INTRODUÇÃO}

O trabalho e o trabalhador estão ambos atravessando um período de mutações aceleradas. Nos últimos dois séculos, a força de trabalho migra do trabalhador braçal para o intelectual. Uma parcela cada vez maior da força de trabalho de todos os países desenvolvidos não trabalha mais com as mãos, mas com ideias, abstrações e teorias. Não produzem objetos físicos e sim saberes e informações (DRUCKER, 2002).

Autores como Toffler (1990), DeMasi (2000), Stewart (1998), entre outros, atentam para o surgimento da sociedade do conhecimento. Observando que, com o surgimento da sociedade do conhecimento, tem-se uma revolução tão profunda quanto a que se viu há dois séculos, quando, em alguns países, totalmente agrícolas, apareceram as primeiras indústrias. Atualmente, em alguns países totalmente industriais, prevalecem atividades terciárias, sobretudo as criativas, levando a novas concepções de trabalho.

As consequências dessas mudanças se projetam por toda a sociedade e suas organizações, que dependem cada vez mais da inovação e do conhecimento. Nesse contexto, os colaboradores, a informação e o conhecimento passam a ser considerados fatores críticos para o sucesso empresarial (SVEIBY, 1998), sendo o gerenciamento dos processos relacionados com o conhecimento nas organizações um dos temas discutidos acaloradamente na última década, pois, tanto os profissionais quanto os acadêmicos afirmam que esses processos são cruciais para se criar e manter uma vantagem competitiva na era pósindustrial.

$\mathrm{Na}$ sociedade do conhecimento, a competitividade é sinônimo de competição acrescida de competência, e a empresa de sucesso é aquela que sabe gerir de forma efetiva as dimensões: pessoas, resultados, inovação e flexibilidade (ABREU, 2002). Nesse contexto, são demandados pelas organizações profissionais que desenvolvem atividades relacionadas à gestão do conhecimento, que, segundo Abreu (2002), nasce como resposta das organizações contemporâneas ao ambiente competitivo.

Profissionais com essa especificidade necessitam de uma sólida formação acadêmica para que possam desenvolver seus serviços no mercado cada vez mais exigente e competitivo. Surge, assim, a necessidade de se desenvolver uma proposta de curso de formação profissional voltada para atender às demandas das organizações por profissionais do conhecimento. Diante do exposto, o presente artigo tem o objetivo de desenvolver uma proposta de formação profissional no nível de Especialização em Gestão Estratégica do Conhecimento.

Para o alcance desse objetivo, fez-se uma análise sobre a demanda, pelas organizações, de profissionais que desenvolvam processos que envolvam a gestão do conhecimento. Apresenta-se uma discussão sobre habilidades necessárias ao profissional envolvido com gestão do conhecimento, traçase um panorama dos cursos de formação dos profissionais do conhecimento no Brasil, e, partindo de uma análise das informações levantadas, são identificadas habilidades necessárias para o profissional que irá atuar com a gestão do conhecimento, que, por sua vez, irão fundamentar o desenvolvimento da matriz curricular do curso proposto.

\section{AS ORGANIZAÇÕES E OS GESTORES DO CONHECIMENTO}

O estudo do conhecimento humano é tão antigo quanto a própria história do homem. Tem sido tema de áreas como psicologia, educação, filosofia e, mais recentemente, das ciências socioeconômicas. Observa-se a importância de se distinguir conceitos relacionados a dados e informações. Dados e informações são menos que conhecimento; de fato, conectam-se entre si para formarem conhecimento. Os dados e informações são como peças de um mosaico, mas não são o desenho final, que, no caso, seria o conhecimento (STEWART, 1998).

Os dados representam um conjunto de fatos discretos sobre um acontecimento. As 
informações acrescentam significado aos dados, "dão forma" - grifo dado pelo autor - e moldam o entendimento das pessoas que as recebem. As informações podem ser dados acrescidos de um contexto, categorizados, calculados, corrigidos, condensados e podem ser apresentados de inúmeras maneiras (STEWART, 1998). Por sua vez, o conhecimento é uma mistura de experiências, valores, contexto compartilhado e discernimento especializado, que fornece uma estrutura para avaliar e incorporar novas experiências e informações que derivam de mentes individuais, mas pode ser apresentado em documentos, tecnologia e processos organizacionais, sendo mais profundo e rico do que a informação, pois está diretamente associado à ação (STEWART, 1998). O conhecimento é avaliado pelas decisões tomadas com base nele e é analisado com base nos resultados dessas decisões. Portanto, enquanto as informações e os dados podem ser avaliados quanto à sua exatidão e inteireza, o conhecimento é avaliado quanto à sua relevância na tomada de decisões (STEWART, 1998).

Conhecimento pode ser explícito e/ou tácito, individual e/ou coletivo (SVEIBY, 1998). O conhecimento tácito são as habilidades inerentes a uma pessoa, é subjetivo, um sistema de ideias, percepção e experiência, saber fazer, difícil de ser formalizado. $\mathrm{O}$ conhecimento explícito é aquele que foi formalizado e está disponível em manuais de procedimentos, livros de pintura, por exemplo, e outros meios de transmissão de informação (SVEIBY, 1998).

No atual contexto competitivo da sociedade do conhecimento, as organizações precisam de novos experts. Profissionais preparados para desenvolver ações relacionadas à preservação e ao desenvolvimento do conhecimento específico, em todos os setores e níveis hierárquicos. Preparados para desenvolver o conhecimento organizacional (por meio da educação, formação e comunicação), registrar o mesmo (por meio da tecnologia e sistemas de informação com níveis diferentes de valor agregado) e integrar seu uso estratégico sob uma administração de recursos de informação unificada (ABREU, 2002).

A demanda advinda das organizações por profissionais do conhecimento é impulsionada pela mudança na forma de execução do trabalho, e leva, inevitavelmente, à definição de novas modalidades de trabalho, que, por sua vez, demandam novos tipos de profissionais (SENGE, 1998). Assim, firmamse várias categorias ligadas à gestão do conhecimento. Aos profissionais da informação ditos "tradicionais" (arquivistas, bibliotecários, museólogos, os profissionais dos meios de comunicação de massa, e até os informáticos) se agregam outros, ditos "emergentes", cujas denominações no mercado de trabalho indicam forte interação de habilidades e conhecimentos técnicos, tais como marketing, história, administração, economia entre outros (MARCHIORI, 2002). Esses profissionais realizam atividades que contribuem para que os recursos de informação sejam mais rentáveis e eficazes na tomada de decisões.

$\mathrm{O}$ mercado precisa de profissionais que combinem a capacidade de gerenciamento com conhecimento técnico, que sejam experts na área de atuação, com uma visão holística de negócios e competência na especialidade, aliada a uma cultura geral ampla. Além de tudo, devem ser confiáveis, éticos, criativos e honestos (MARCHIORI, 2002).

Considera-se que os profissionais responsáveis por ações relacionadas à gestão do conhecimento auxiliam no processo de tomada de decisão, sendo capazes de identificar, recuperar, analisar, disseminar e usar a informação relevante e prioritária (FERREIRA, 2003; O'BRIEN, 2010).

Os profissionais que desenvolvem atividades relacionadas à gestão do conhecimento devem possuir uma visão ampla da organização e de seus negócios e uma visão abrangente da tecnologia, compreendendo as dimensões estratégicas e operacionais, os mecanismos de gestão de recursos humanos, tecnológicos, financeiros, materiais e físicos envolvidos. Nesse sentido, acredita-se que os 
profissionais do conhecimento precisam possuir habilidades de visão sistêmica (conceituais), de relacionamento interpessoal e técnicas (MARCHIORI, 2002; FERREIRA, 2003; O'BRIEN, 2010; OLIVEIRA， 2007; FERREIRA; FERREIRA; LETALIEN, 2007).

Os profissionais ligados à gestão do conhecimento organizacional devem possuir habilidades conceituais para visualizar a organização como um conjunto integrado. Sob uma perspectiva sistêmica, entende-se que as organizações funcionam como qualquer ser vivo, importando recursos e matérias primas do meio ambiente, que são convertidos em produtos ou serviços, e que, por seu turno, são exportados para consumo interno ou externo. As habilidades conceituais estão relacionadas à capacidade de se posicionar no ponto de vista da organização, perceber como as várias funções são interdependentes e como a alteração em uma delas afeta todas as demais; têm implicação na capacidade de visualizar a organização dentro do seu ambiente externo e compreender as forças políticas, econômicas, tecnológicas e sociais que atuam sobre ela, saber identificar a relação entre valores organizacionais e a criação do conhecimento; permitem, enfim, não somente reconhecer essas relações, mas destacar os elementos significativos em cada situação e identificar a alternativa mais adequada para o processo que envolve a gestão do conhecimento (MIGUEL e TEIXEIRA, 2009). As habilidades conceituais estão relacionadas aos seguintes aspectos: conhecimento sobre o setor industrial, conhecimento das estruturas de poder da organização, conhecimento sobre os processos de tomada de decisão, conhecimento das forças do mercado que influenciam a empresa, compreensão da cultura organizacional, conhecimento das preferências dos administradores (OLIVEIRA, 2007).

As habilidades de relacionamento interpessoal estão ligadas à capacidade de trabalhar com eficácia como membro de um grupo e de conseguir esforços cooperativos no grupo, na direção dos objetivos definidos; aptidões para trabalhar com pessoas e para obter resultados por meio dessas pessoas; capacidade para criar uma atmosfera de segurança, para comunicar e encorajar a comunicação e para compreender as necessidades e motivações dos membros do grupo em relação às demandas de informação. Estão, assim, associadas às habilidades humanas fatores como: astúcia, comunicação, perspicácia, persistência, intuição, aprendizagem, confiabilidade (OLIVEIRA, 2007).

As habilidades técnicas envolvem conhecimento especializado, habilidade analítica dentro da especialidade e facilidade no uso das técnicas e do instrumental da tecnologia da informação. As habilidades técnicas estão relacionadas ao conhecimento sobre temas como: web 2.0, redes de informação, sistemas gerenciadores de bases de dados, aplicações de microcomputadores e redes, hardware e programação (OLIVEIRA, 2007).

\section{PANORAMA DOS CURSOS DE FORMAÇÃO DO PROFISSIONAL DO CONHECIMENTO}

A exigência de um composto gerencial, a utilização de tecnologias e a importância de se compreender e oferecer a informação com valor agregado indicam, pelo menos, três recortes que já se apresentam como realidades no contexto dos cursos de graduação e pósgraduação em gestão do conhecimento ofertados.

Nos cursos relacionados às Ciências Exatas, destacam-se as áreas científicas da informática, e da matemática, e enfatizam as disciplinas relacionadas às metodologias de engenharia de conhecimento, como CommonKADS, entre outras, na área da Engenharia de Ontologias e PSM. Sob esse enfoque, o conhecimento é visto como um recurso a ser otimizado via diferentes arquiteturas de hardware, software e de redes de telecomunicações adequadas aos diferentes sistemas de informação - em especial, aos empresariais (MARCHIORI, 2002). No escopo das Ciências Sociais aplicadas à gestão do conhecimento, tem-se como objetivo o desenvolvimento da competitividade empresa- 
rial e os processos ligados à inovação. Os cursos de Administração de Empresas estão capacitando os profissionais para administração de tecnologias da informação em sintonia com os objetivos empresariais. A intenção é formar um profissional que, além das tradicionais disciplinas da área, domine o planejamento e uso estratégico das tecnologias da informação e as especificações de qualidade e segurança da informação empresarial. É também nesse contexto que se intensificam as propostas de estudos em gestão do conhecimento (MARCHIORI, 2002). O terceiro enfoque é o da Ciência da Informação (INSTITUTE OF INFORMATION SCIENTISTS, 2010). Uma formação profissional em gestão do conhecimento, sob essa óptica, volta-se para o contexto social onde há fornecimento e demandas de informação, assim como a necessidade do gerenciamento de recursos de informação. Sob o enfoque da Ciência da Informação, a formação do profissional tem caráter interdisciplinar, incluindo aspectos da Ciência da Computação, mas também incluindo um grande número de outras áreas, como: Biblioteconomia, Comunicações, Direito, Matemática, Gestão Empresarial, Ciências Cognitivas, Ciências Sociais e Políticas Públicas. Diante do exposto, entendese que o enfoque da Ciência da Informação engloba os demais.

\section{PROJETO DO CURSO DE ESPECIALIZAÇÃO EM GESTÃO ESTRATÉGICA DO CONHECIMENTO}

Na elaboração de uma proposta de curso de Especialização em Gestão Estratégica do Conhecimento, diversos aspectos devem ser considerados:

1. é uma área aberta a novas carreiras e atividades diversificadas;

2. é direcionada pela aplicação de novas tecnologias;

3. é multidisciplinar;
4. está sofrendo uma mudança de paradigma, na medida em que a informação e o conhecimento transformam-se em ativos estratégicos e valiosos para as organizações (CIANCINI, 1991).

Assim, a formação de especialistas em gestão estratégica do conhecimento persegue os seguintes objetivos: a) formação de profissionais qualificados, criativos e críticos, na perspectiva da construção de uma sociedade justa e democrática; b) o desenvolvimento de atividades multidisciplinares, que agreguem competências técnicas específicas, uma visão sistêmica sobre o processo de codificação, gestão e disseminação do conhecimento (tácito ou explícito), de maneira a formar profissionais mais capacitados na construção de uma sociedade que vise ao bem comum da sociedade; c) a formação de profissionais capacitados à implementação de modelos, métodos e técnicas de gestão e disseminação dos conhecimentos (explícitos e tácitos) em organizações, públicas e privadas, e na sociedade em geral; e, d) o avanço do conhecimento científico e tecnológico.

$\mathrm{O}$ curso tem como público-alvo os pesquisadores e profissionais responsáveis pela codificação do conhecimento organizacional, e os responsáveis pela utilização do conhecimento como fator de produção estratégico no gerenciamento de negócios relacionados à economia baseada no conhecimento.

Sugere-se que um curso de Especialização em Gestão Estratégica do Conhecimento deva, em sua matriz curricular, contemplar disciplinas que contribuam para desenvolvimento das habilidades de visão sistêmica (conceituais), interpessoais e técnicas. O conjunto das disciplinas apresentadas no Quadro 1, a seguir, é dividido em três blocos: disciplinas relacionadas ao desenvolvimento de habilidades conceituais, disciplinas relacionadas ao desenvolvimento de habilidades interpessoais e outro rol de disciplinas para o desenvolvimento de habilidades técnicas. 
Quadro 1: Matriz curricular proposta para o curso de Especialização em Gestão Estratégica do Conhecimento - GEC.

\begin{tabular}{|c|c|c|}
\hline Conceituais & Interpessoais & Técnicas \\
\hline $\begin{array}{c}\text { Gestão Empresarial e } \\
\text { Competitividade }\end{array}$ & Cultura e clima organizacional & $\begin{array}{c}\text { Inteligência Computacional e Data } \\
\text { Mining }\end{array}$ \\
\hline $\begin{array}{c}\text { Planejamento e Estratégia } \\
\text { Organizacional }\end{array}$ & Gestão de Recursos Humanos & $\begin{array}{c}\text { Gestão da Tecnologia e Segurança da } \\
\text { Informação }\end{array}$ \\
\hline $\begin{array}{c}\text { Inteligência Competitiva } \\
\text { Fundamentos de Gestão do } \\
\text { Conhecimento }\end{array}$ & Gestão da Inovação & $\begin{array}{c}\text { Sistemas de Informação e Suporte à } \\
\text { Decisão }\end{array}$ \\
\hline $\begin{array}{c}\text { Organizações Intensivas em } \\
\text { Conhecimento }\end{array}$ & Gestão da Mudança & $\begin{array}{c}\text { Fundamentos da Engenharia do } \\
\text { Conhecimento }\end{array}$ \\
\hline $\begin{array}{c}\text { Aprendizagem Organizacional } \\
\text { Comunicação }\end{array}$ & $\begin{array}{c}\text { Tecnologia da Informação e } \\
\text { Desenvolvimento Humano e Gestão }\end{array}$ & $\begin{array}{c}\text { Métodos e Técnicas de Engenharia } \\
\text { do Conhecimento }\end{array}$ \\
\hline $\begin{array}{c}\text { Desafios Futuros da Gestão do } \\
\text { Conhecimento nas Organizações }\end{array}$ & $\begin{array}{c}\text { Liderança em Organizações } \\
\text { Intensivas em Conhecimento } \\
\text { Estratégica do Conhecimento }\end{array}$ & $\begin{array}{c}\text { Ambientes Computacionais } \\
\text { Distribuídos para Engenharia do } \\
\text { Conhecimento }\end{array}$ \\
\hline $\begin{array}{c}\text { Seminários Avançados em Gestão } \\
\text { Conhecimento }\end{array}$ & \begin{tabular}{c} 
Gestão de Custos \\
\hline
\end{tabular}
\end{tabular}

Disciplinas relacionadas às habilidades conceituais abordam de forma abrangente os ambientes interno e externo das organizações. As disciplinas "Gestão Empresarial e Competitividade", e "Planejamento e Estratégia Organizacional" são orientadas para a disseminação dos conceitos relacionados à importância dos processos de gestão na criação da vantagem competitiva, no contexto da sociedade do conhecimento. As disciplinas "Inteligência Competitiva", "Fundamentos da Gestão do Conhecimento", "Organizações Intensivas em Conhecimento" e "Aprendizagem Organizacional" visam a dar uma visão geral da história da gestão do conhecimento; identificar as suas etapas-chave; determinar um quadro de referência e uma linguagem clara para os conceitos de conhecimento e de gestão do conhecimento; definir os conceitos-chave da gestão de conhecimento, tais como: capital intelectual (ativos intangíveis), aprendizagem e memória organizacional, taxonomia do conhecimento e comunidades de prática, por meio da análise de conceitos; e também descrever os principais papéis e responsabilidades exigidos para aplicações de gestão do conhecimento. As disciplinas "Seminários Avançados em Gestão Estratégica do Conhe- cimento" e "Desafios Futuros da Gestão do Conhecimento nas Organizações" buscam promover a reflexão e o posicionamento crítico e ético sobre os processos de gestão do conhecimento.

Para o desenvolvimento de habilidades interpessoais, optou-se por um leque de disciplinas que contribuam para a formação de um profissional que compreenda a importância das pessoas no processo de gestão do conhecimento. Assim, as disciplinas "Cultura e Clima Organizacional", "Gestão de Recursos Humanos", "Criatividade" e "Gestão da Mudança" abordam questões ligadas à gestão e monitoração dos ativos intangíveis das organizações, à comunicação interpessoal, contextualização do capital intelectual e suas relações com o processo de inovação, além de tratar dos indicadores e estratégias de potencialização do valor do conhecimento nas organizações. As disciplinas "Desenvolvimento Humano e Gestão", "Liderança em Organizações Intensivas em Conhecimento" e "Desafios Futuros do Profissional do Conhecimento" têm como foco o estudo sobre a evolução do conceito de "gestão de pessoas", a inteligência interpessoal, a compreensão da natureza do comportamento e do trabalho do 
líder organizacional, e, ainda, uma reflexão sobre os desafios dos profissionais na sociedade do conhecimento.

No desenvolvimento de habilidades técnicas, optou-se por um rol de disciplinas que capacitem o profissional para atuar e gerenciar questões específicas, particularmente aquelas relacionadas à gestão de custos e ao relacionamento entre a tecnologia da informação e a gestão do conhecimento. Essa gama de disciplinas apresenta conceitos básicos sobre as tecnologias da informação (TI), aplicações de sistemas de informação na gestão do conhecimento, além de questões relacionadas à gestão de ativos financeiros na implantação de programas de gestão do conhecimento. Com excessão da disciplina "Gestão de Custos", que tem um enfoque administrativo e gerencial, as demais se voltam para o estudo dos processos da engenharia do conhecimento: identificação, aquisição, formalização, representação, distribuição, implementação, avaliação e manutenção. Visam também ao entendimento e à aplicação das principais metodologias da engenharia do conhecimento, técnicas $\mathrm{e}$ metodologias de modelagem de sistemas de gestão do conhecimento organizacional (ex. Desire, Protégé, Vital, MIKE, Task), de metodologias como CommonKADS, bem como o estudo de casos práticos e reais de sistemas de conhecimento em organizações.

\section{CONSIDERAÇÕES FINAIS}

A partir da discussão sobre a demanda de profissionais do conhecimento, da apresentação das habilidades requeridas desses profissionais e da oferta de formação acadêmica, foi desenvolvida uma proposta de formação profissional no nível de Especialização em Gestão Estratégica do Conhecimento.

Do profissional que desenvolve atividades voltadas à gestão do conhecimento organizacional exige-se permanente aprendizado. Seu campo de atuação é muito vasto e requer conhecimentos diversificados, o ambiente muda, a ciência e a tecnologia evoluem, os processos se alteram e tudo isso requer permanente atualização dos conhecimentos. Nesse contexto, quanto mais se sabe, mais rapidamente se aprende coisas novas. As mudanças não surgem do nada, muitas novidades aparentes utilizam os mesmos princípios básicos e conceitos que existem e são adotadas há várias décadas, ou mesmo há vários séculos. A capacidade de fazer analogias e assimilar conhecimentos novos é diretamente proporcional ao volume de conhecimentos possuídos. Na sociedade do conhecimento, na qual convivemos com grandes mudanças, os profissionais que atuam no processo de gestão estratégica da informação devem estar cientes de que a aprendizagem é um processo contínuo e permanente. Os profissionais que atuam na gestão do conhecimento precisam entender a importância da formação continuada em uma sociedade que vive em acelerado processo de mudança. $\mathrm{O}$ profissional do futuro é responsável por sua formação e deve adquirir o hábito da aprendizagem continuada (DRUCKER, 2002). Nesse sentido, recomenda-se um diálogo frequente entre as organizações e a academia, para que os cursos ofertados venham ao encontro das necessidades demandas pelo mercado e, nesse contexto, acredita-se pertinente o projeto do curso de Especialização em Gestão Estratégica do Conhecimento.

\section{REFERÊNCIAS}

ABREU, A. F. Gestão da inovação: uma nova abordagem orientada à gestão corporativa. Working Paper, IGTI, 2002.

CIANCINI, R. B. A Gerência da informação: mudança nos perfis profissionais. Ci. Inf., Brasília, DF, v. 20, n. 2, p. 204-208, jul./dez. 1991.

DRUCKER P. F. Introdução à administração. São Paulo: Pioneira Thomson, 2002.

DEMASI, D. O futuro do trabalho: fadiga e ócio na sociedade pós-industrial. 5. ed. Rio de Janeiro: José Olympio; Brasília, DF: Ed. da UnB, 2000.

FERREIRA，D. T. Profissional da informação: perfil de habilidades demandadas pelo mercado de trabalho. Ci. Inf., Brasília, DF, v. 32, n. 1, abr. 2003. 
FERREIRA, F.; FERREIRA, F.; LETALIEN, B. L. Information professionals in Brazil: core competencies and professional development. Information Research, v. 12, jan. 2007.

INSTITUTE OF INFORMATION SCIENTISTS. Disponível em <http://www.iis.org.uk/>. Acesso em: ago. 2010.

MARCHIORI, P. Z. A ciência e a gestão da informação: compatibilidades no espaço profissional. Ci. Inf., Brasília, DF, v. 31, n. 2, ago. 2002.

MIGUEL, L. A. P.; TEIXEIRA, M. L. M. Valores organizacionais e criação do conhecimento organizacional inovador. RAC. Curitiba, jan./mar. 2009.

O'BRIEN, J. A. Sistemas de informação e as decisões na Era da Internet. São Paulo: Saraiva, 2010.
OLIVEIRA, P.; LACERDA, J. Habilidades e competências desejáveis aos profissionais de inteligência competitiva. Ci. Inf., Brasília,DF, v. 36, n. 2, ago. 2007.

SENGE, P. A quinta disciplina: arte e prática da organização de aprendizagem 2. ed. São Paulo: Editora Best Seller; Círculo do Livro, 1998.

STEWART, T. A. Capital intelectual: a nova vantagem competitiva nas empresas. 8. ed. Tradução de Ana Beatriz Rodrigues, Priscilla Martins Celeste. Rio de Janeiro: Campus, 1998.

SVEIBY, K. E. A nova riqueza das organizações. Rio de Janeiro: Campus, 1998.

TOFFLER, A. Powershift: as mudanças do poder. Rio de Janeiro: Record, 1990.
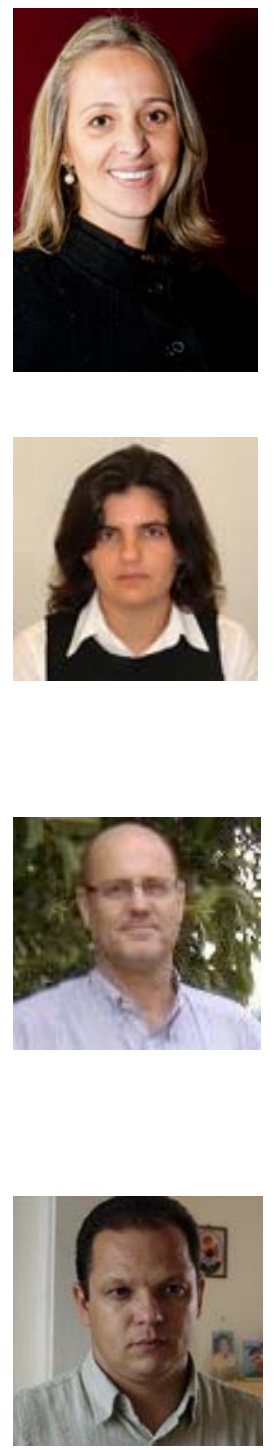

\section{DADOS DOS AUTORES}

Ana Paula Lisboa Sohn - Doutoranda no Programa de Pós Graduação em Engenharia de Produção na Universidade Federal de Santa Catarina, com doutorado Sanduíche na Universidade do Minho em Portugal. Mestre em Relações Internacionais para o Mercosul pela Universidade do Sul de Santa Catarina (2004). Especialista em Gestão de Empresas pela Universidade Federal de Santa Catarina (1998) Graduada em Pedagogia pela Universidade do Estado de Santa Catarina (1993), e Administração pela Universidade Federal de Santa Catarina (1996). Desde agosto de 2000 é professora titular da Universidade do Vale do Itajaí em cursos de graduação e pós graduação.

Filipa Dionísio Vieira - Licenciada em Engenharia de Producão - Ramo Têxtil pela Universidade do Minho, em 1993, Mestre em Comércio Internacional, pela Escola de Economia e Gestão da Universidade do Minho, em 1998 e Doutora em Producão e Sistemas, pela Escola de Engenharia da Universidade do Minho, na área de Engenharia Económica, em 2007. Professora Auxiliar do Departamento de Producão e Sistemas da Escola de Engenharia da Universidade do Minho. Investigadora do CGIT - Centro de Investigaçao de Gestão Industrial e da Tecnologia.

Nelson Casarotto Filho - Possui graduação em Engenharia Química pela Universidade Federal do Rio Grande do Sul (1974), mestrado em Engenharia de Produção pela Universidade Federal de Santa Catarina (1977) e doutorado em Engenharia de Produção pela Universidade Federal de Santa Catarina (1995) com sanduíche na Universidade do Minho - Portugal. Professor Associado da Universidade Federal de Santa Catarina, onde pesquisa no Laboratório de Sistemas de Apoio ao Desenvolvimento de Projetos e Investimentos (LABSAD), ligado ao Departamento de Engenharia de Produção e Sistemas.

João Artur de Souza - Graduação em Matemática (Licenciatura) pela Universidade Federal de Santa Catarina (1989), mestrado em Matemática e Computação Científica pela Universidade Federal de Santa Catarina (1993), doutorado em Engenharia de Produção pela Universidade Federal de Santa Catarina (1999) e pós-doutorado pela Universidade Federal de Santa Catarina (2000). Professor Associado da Universidade Federal de Santa Catarina do Departamento de Engenharia do Conhecimento. 